\title{
Phenothiazine-benzimidazole architecture as an efficient interfacial charge transport layer for perovskite blue light emitting diodes
}

\author{
Keval K. Sonigara, ${ }^{\mathrm{a}}$ Hailong Wang, ${ }^{\mathrm{c}, \mathrm{d}}$ Jiang Chao, ${ }^{\mathrm{b}}$ Zhiyuan Xie, ${ }^{\mathrm{c}, \mathrm{d}, *}$ Jian Fan, ${ }^{\mathrm{b}, *}$ Jingsong Huang, ${ }^{\mathrm{a}, *}$ Paul \\ N Stavrinou, ${ }^{\mathrm{a}, \mathrm{e}, *}$ Donal D C Bradley ${ }^{\mathrm{a}, \mathrm{f}, *}$ \\ ${ }^{a}$ Oxford Suzhou Centre for Advanced Research (OSCAR), University of Oxford, Building A, 388 Ruo Shui Road, \\ Suzhou Industrial Park, Jiangsu, 215123, P.R. China \\ ${ }^{b}$ Institute of Functional Nano \& Soft Materials (FUNSOM), Jiangsu Key Laboratory for Carbon-Based Functional \\ Materials \& Devices, Soochow University, Suzhou, Jiangsu 215123, China \\ 'State Key Laboratory of Polymer Physics and Chemistry, Changchun Institute of Applied Chemistry, Chinese \\ Academy of Sciences, Changchun 130022, P. R. China \\ ${ }^{\mathrm{d}}$ University of Science and Technology of China, Hefei 230026, P. R. China \\ eDepartment of Engineering Science, University of Oxford, Oxford OX1 3PJ, U.K. \\ fPhysical Science and Engineering Division, King Abdullah University of Science and Technology, Thuwal, 23955- \\ 6900 Kingdom of Saudi Arabia \\ * Corresponding authors
}

\begin{abstract}
Solution-processed metal halide perovskite light-emitting diodes (Pe-LEDs) show great promise in a range of optoelectronic applications. However, these devices can be limited by poor interfaces to the perovskite films due to poor crystallization control during film processing caused by de-wetting from the bottom layer. The deep (Highest Occupied Molecular Orbital) HOMO levels of the emitters also lead to large charge injection barriers for standard electrodes. To improve this, we develop and report on a small molecule, BPS2, based on phenothiazinebenzimidazole with Lewis base sites. This promising interfacial material is then applied to blue PeLEDs where the energy band alignment of BPS2 to the blue perovskite emitter helps to reduce the hole-injection barrier while blocking electrons. BPS2 can be solution-processed with non-chlorinated organic solvents and provides improved wettability towards perovskite precursor solutions compared to conventional PEDOT:PSS hole transport films. A thin interlayer of BPS2 introduced between PEDOT:PSS and a perovskite emission layer is shown to improve both the device external quantum efficiency and luminance in comparison to the reference device without the interlayer.
\end{abstract}

Keywords: Solution processed small molecule interlayer, hole injection interlayer, interface improvement, charge recombination barrier improvement, perovskite light emitting diodes.

\section{INTRODUCTION}

Perovskite light-emitting diodes (PeLEDs) and transistors represent an important family of next-generation optoelectronic devices.[1, 2] However, the current state-of-the-art blue-emitting PeLEDs require improvement for commercial application.[3] Recent research on the subject has explored various strategies ranging from halogen regulation to using quasi-2D and quantum dot structures in the perovskite emission layer.[4] Outside the development of the active layers, interest has also turned to the ancillary layers in the PeLED device, such as the hole- and electron-injection layers. One of the most commonly used hole-injecting layers (HIL) is poly(3,4ethylenedioxythiophene):poly(styrenesulfonate) (PEDOT:PSS), which offers high charge carrier mobility, good transparency, and excellent processability.[5] For the case of PeLEDs, however, the aqueous processing solvent and acidic nature of PEDOT:PSS frequently cause problems for adjacent layers formed from perovskite precursor solutions. Furthermore, from an electronic point of view, a key difficulty is the large hole injection barrier between the HOMO levels of PEDOT:PSS (4.9-5.2 eV) and perovskite (5.6-6.1 eV).[5]

Attempts to address this interface issue follow two main approaches: i) adding surfactants, metal salts and/or copolymer components to the PEDOT:PSS solution to create a blend film and ii) introducing interlayers, to reduce the energy barrier and/or to achieve better surface energy of bottom layer for superior perovskite film formation.[6] Several polymer-based interlayers have been successfully demonstrated for this role, including, PVK, Poly-TDP and 
TFB:PFI.[6] Nevertheless, the resulting surface properties, which typically lead to dewetting issues with respect to the perovskite precursors, frequently limit the film-formation capability. Surprisingly, there have been few reports to date from research groups utilizing small molecules as interlayers. Small molecules have been reported to improve device performance through their blending in polymeric charge transport layers and/or in the emission layers realizing passivation and crystal growth control.[6] The present work introduces phenothiazine donor-based small molecules for use as an interlayer between PEDOT:PSS and blue-emitting Rb-Cs mixed alloy perovskite active layers. The two new materials are: i) BPS with a classical non-extended core and, ii) BPS2 with an extended symmetrical branch for improved solubility and film processability (Figure 1). The HOMO levels for both are higher than PEDOT:PSS and better aligned with the active perovskite material, leading to a lower hole injection barrier and their LUMO (lowest unoccupied molecular orbital) levels provide a significant barrier to electrons. In addition, the excellent solubility of BPS2 in chlorine-free solvents (e.g. Toluene) allows formation of high-quality thin films directly upon PEDOT:PSS that exhibit improved surface energy compatibility with the perovskite precursor solvent DMSO. Compared to a reference device, incorporation of a BPS2 interlayer increased the external quantum efficiency (EQE), with a corresponding enhancement in luminance. Both of these improvements can be attributed to improved interfacial properties and, in part, the perovskite active layer structure.
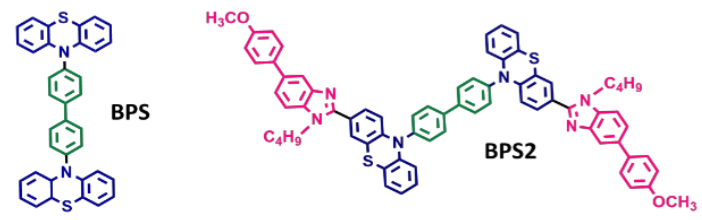

Figure 1. Chemical structures of new interfacial hole transporting materials. Green: biphenyl (BP) accepter (A); Blue: Phenothiazine (PTZ) donor (D); Pink: benzimidazolium (BM) pseudo acceptor (A') and methoxy phenyl (MOP) donor (D')

\section{EXPERIMENTAL}

Ultraviolet-visible (UV-Vis) absorption and Photoluminescence (PL) spectra were measured for thin films coated on glass substrates using a Shimadzu 4200 UV-Vis spectrophotometer and Hitachi F-4700 fluorescence spectrophotometer, respectively. Three-electrode cyclic voltammetry (CV) measurements used a Biologic SP200 electrochemical workstation; thin films of BPS/BPS2 were spin coated (BPS: $5 \mathrm{mg} / \mathrm{mL}$ in hot chlorobenzene, 4000 $\mathrm{rpm}, 30$; BPS2: $5 \mathrm{mg} / \mathrm{mL}$ in Toluene, $4000 \mathrm{rpm}, 30 \mathrm{~s})$ on an ITO $\left(2.5 \mathrm{~cm} \mathrm{x} 1 \mathrm{~cm}\right.$, strip, active area $\left.1 \mathrm{~cm}^{2}\right)$ working electrode. The non-aqueous reference electrode was freshly filled with $0.1 \mathrm{M} \mathrm{AgNO}_{3}$ and $0.1 \mathrm{M}$ tetrabutylammonium hexafluorophosphate $\left(\mathrm{TBAPF}_{6}\right)$ in acetonitrile and externally standardized with a Ferrocene/Ferrocenium $\left(\mathrm{Fc} / \mathrm{Fc}^{+}\right)$ reference. Pt-wire served as the counter electrode. HOMO values were determined from the first oxidation potential onset, $E_{\text {onset }}$, vs $\mathrm{Ag} / \mathrm{Ag}^{+}$observed in the voltammogram via $E_{\text {Номо }}=-\left(5.1+\left(E_{\text {onset }}-E_{F c / F c}{ }^{+}\right)\right) \mathrm{eV}$. [7] In our case, $\mathrm{Fc} / \mathrm{Fc}^{+}$redox potential is $0.215 \mathrm{~V} \mathrm{Vs} \mathrm{Ag} / \mathrm{Ag}^{+}$in reference system. A standard sessile drop apparatus (Kruss DSA25E) was used for contact angle measurements on thin film samples coated on glass substrates. PeLED current-density $v s$ voltage $(J-V)$ and luminance $v s$ voltage $(L-V)$ characteristics were measured using a Keithley 2400 source meter and a calibrated silicon photodiode.

BPS was synthesized by a Buchwald-Hartwig reaction between biphenyl halide and phenothiazine. BPS2 Synthesis route follows a multi-step synthesis method directly from BPS with slight change in reaction protocol described in reported earlier elsewhere.[8] That include formylation, coupling with 4-Br-orthophenylene diamine, substitution of hydrogen by butyl chain from benzimidazole derivative, and Suzuki coupling with phenyl boronic acid. (BPS) 10(4'-(4a,10a-dihydro- 10H-phenothiazin-10-yl)-[1,1'-biphenyl]-4-yl)-10H-phenothiazine: (Yield, $4.5 \mathrm{gm}, 85 \%) .{ }^{1} \mathrm{H}$ NMR $\left(400 \mathrm{MHz}, \mathrm{CDCl}_{3}\right) \delta: 7.88(\mathrm{~d}, J=8.1 \mathrm{~Hz}, 4 \mathrm{H}), 7.50(\mathrm{~d}, J=8.1 \mathrm{~Hz}, 4 \mathrm{H}), 7.07(\mathrm{dd}, J=7.4,1.8 \mathrm{~Hz}, 4 \mathrm{H}), 6.95-$ $6.82(\mathrm{~m}, 8 \mathrm{H}), 6.36(\mathrm{~d}, J=8.1 \mathrm{~Hz}, 4 \mathrm{H})$. (BPS2)4,4'-bis(3-(1-butyl-5-(4-methoxyphenyl)-1H-benzo[d]imidazol-2-yl)10H-phenothiazin-10-yl)-1,1'-biphenyl: (Yield: $0.22 \mathrm{~g}, 71 \%) .{ }^{1} \mathrm{H}$ NMR (400 MHz, DMSO-D $) ~ \delta: 8.15$ (d, $J=8.1$

$\mathrm{Hz}, 4 \mathrm{H}), 7.83(\mathrm{~d}, J=7.4 \mathrm{~Hz}, 2 \mathrm{H}), 7.67(\mathrm{q}, J=9.5,9.1 \mathrm{~Hz}, 10 \mathrm{H}), 7.54(\mathrm{~s}, 2 \mathrm{H}), 7.53-7.45(\mathrm{~m}, 2 \mathrm{H}), 7.42(\mathrm{~d}, J=8.7$ $\mathrm{Hz}, 2 \mathrm{H}), 7.16(\mathrm{~d}, J=7.4 \mathrm{~Hz}, 2 \mathrm{H}), 7.03(\mathrm{q}, J=7.2 \mathrm{~Hz}, 6 \mathrm{H}), 6.94(\mathrm{t}, J=7.4 \mathrm{~Hz}, 2 \mathrm{H}), 6.42(\mathrm{~d}, J=8.6 \mathrm{~Hz}, 2 \mathrm{H}), 6.30$ $(\mathrm{d}, J=8.3 \mathrm{~Hz}, 2 \mathrm{H}), 4.40-4.25(\mathrm{~m}, 4 \mathrm{H}), 3.80(\mathrm{~d}, J=2.7 \mathrm{~Hz}, 6 \mathrm{H}), 1.69(\mathrm{~s}, 4 \mathrm{H}), 1.19(\mathrm{~d}, J=6.8 \mathrm{~Hz}, 4 \mathrm{H}), 0.81(\mathrm{t}, J=$ $7.3 \mathrm{~Hz}, 6 \mathrm{H})$. 


\section{RESULTS AND DISCUSSION}

Photophysical and electrochemical measurements were performed for solid films coated on glass and ITO substrates respectively. Figure 2(a) displays the absorbance and PL spectra. Absorbance is dominated by a high energy peak around $270-275 \mathrm{~nm}$, arising from strongly localized aromatic $\pi-\pi^{*}$ transitions. The longer wavelength feature, near $325 \mathrm{~nm}$, likely involves transitions between spatially delocalised HOMO and LUMO electron distributions for BPS. In the case of BPS2, an additional lower energy feature is seen, around $375 \mathrm{~nm}$, likely arising from the extended conjugation within the BM-MOP unit. Energy gap values may be estimated from the onset of the absorbance spectra and give $3.06 \mathrm{eV}$ and $2.75 \mathrm{eV}$ for BPS and BPS2, respectively. The smaller gap for BPS2 is also consistent with the $\sim 86 \mathrm{~nm}$ red-shifted PL peak relative to BPS. Assessing the intramolecular charge transfer character for these states will require further investigation. The first oxidation potential in Figure 2(b) was used to determine the HOMO energy level; the observed secondary oxidation peaks at high potential are expected to be due to di-cation formation. Figure 2(c) shows a schematic energy level diagram for the materials used in the blue emitting PeLED device structures. It is clear from Figure 2(c) that the HOMO levels for both interlayer materials sit between that of PEDOT:PSS and the perovskite valence band. Significantly, their respective LUMO levels are also expected to be sufficient to block unwanted electron leakage into the PEDOT:PSS layer. These relative alignments bode well for inclusion of the interlayers in the intended blue-emitting PeLED structures.
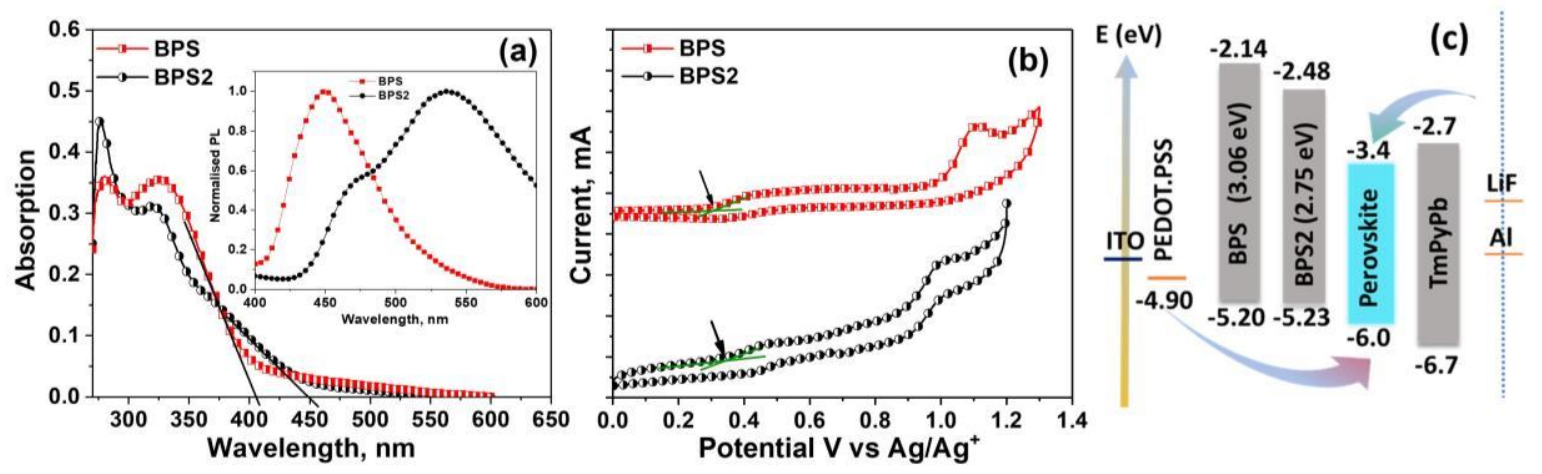

Figure 2 (a) UV-vis absorbance and PL emission (Inset) spectra of thin films coated on glass. Thin black lines superimposed on the absorption spectra indicate the long-wavelength optical absorption edge. (b) cyclic voltammetry curves (scanned at $50 \mathrm{mV} / \mathrm{s}$ ) for solid films coated on ITO substrates. The potential, $V$, is measured relatve to a $\mathrm{Ag} / \mathrm{Ag}^{+}$electrode in acetonitrile in the presence of $0.1 \mathrm{M}$ TBAPF6; the black arrows indicate the first oxidation potentials deduced from the change in current slope, (c) Schematic Energy level diagram for the materials used in our blue emitting PeLEDs.

The wettability of the perovskite precursor solution on the bottom layer can dramatically affect the perovskite crystal growth and the subsequent film quality with improved wetting linked to higher quality perovskite films.[6] Figure 3 shows the contact angle for dimethyl sulfoxide (DMSO) solvent droplets on thin films $(<30 \mathrm{~nm})$ of PEDOT:PSS, BPS and BPS2 coated onto glass substrates. For the BPS2 surface, the contact angle is around 30\% smaller than found for PEDOT:PSS and BPS surfaces, suggesting a greater wettability for polar solutions thereon. We suggest that the BPS2 non-planer geometry, with both butyl chains and methoxy groups plays an important role. In contrast, BPS is expected to have a rigid structure in the solid state as biphenyl ring torsion is frustrated. Highly uniform BPS2 films are readily obtained from room temperature solutions whereas for BPS $60-70{ }^{\circ} \mathrm{C}$ pre-heating is required. With these results we have focused our PeLED fabrication efforts on BPS2 containing devices (see Figure 4(a)). BPS2 was introduced as a very thin interlayer $(\leq 10 \mathrm{~nm})$ between the PEDOT:PSS and blue perovskite ( $\mathrm{RbCsBrCl})$ emitter. 

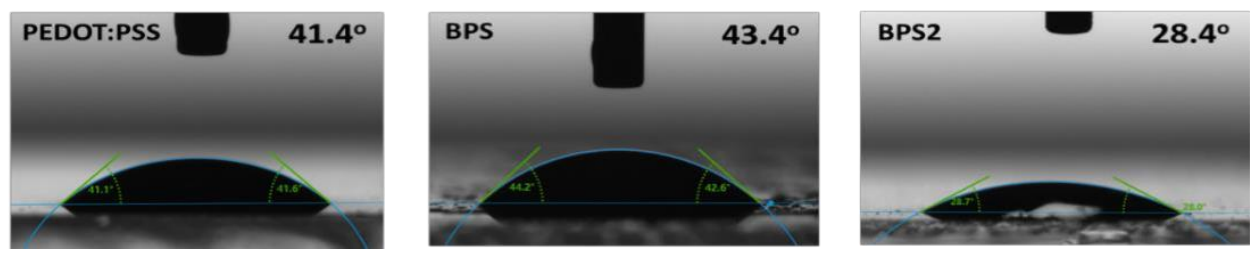

Figure 3. Contact angle measurements, after 5 seconds dwell time, for DMSO on PEDOT:PSS (left), BPS (middle) and BPS2 (right).

The current density vs voltage characteristics of Figure 4(b) compare devices with and without the BPS2 interlayer. Both traces exhibit similar diode characteristics with a turn-on voltage $(\sim 2.8 \mathrm{~V})$ slightly larger than the $2.6 \mathrm{eV}$ bandgap of the blue-emitting $\mathrm{RbCsBrCl}$ perovskite. Compared to the reference structure without the BPS2 interlayer (see Figures 4(b-c)), there are significant improvements in luminance, especially at lower voltages and in external quantum efficiency. Two underlying reasons are highlighted. The first is the better energy level alignment of BPS2 with the blue emitter (Figure 2(c)), facilitating hole injection from and limiting electron leakage to the PEDOT:PSS/ITO contact. The second relates to a more favorable surface energy compatibility with the perovskite precursor solution (Figure 3), which should result in an improved film structure, reducing charge carrier recombination at the interface. The results confirm that introduction of a BPS2 interlayer improves key device metrics.

(a)

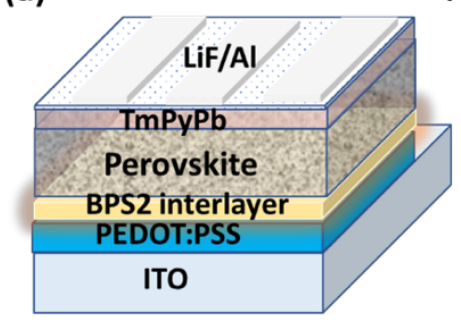

(b)

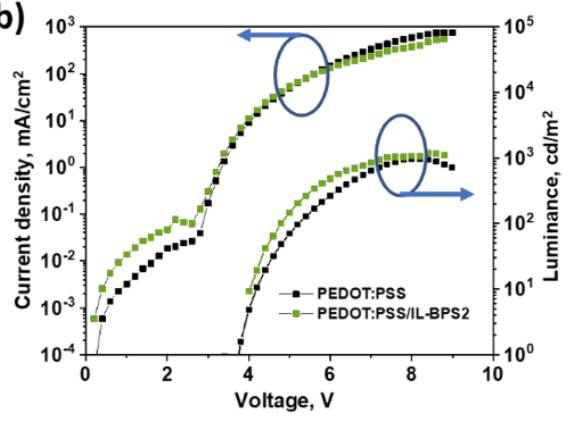

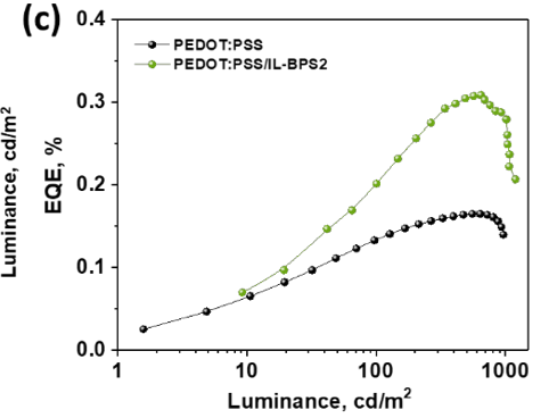

Figure 4. (a) General PeLED device configuration comprising ITO/PEDOT:PSS/Interlayer (< $10 \mathrm{~nm}) /$ Perovskite/ 1,3,5-Tris(3pyridyl- 3-phenyl)benzene $(\mathrm{TmPyPb}) / \mathrm{LiF} / \mathrm{Al}$; (b) Current density and luminance $v s$ voltage characteristics; and (c) EQE $v s$ luminance.

\section{CONCLUSIONS}

We have investigated photophysical and electrochemical properties of a new family of interlayer materials based on phenothiazine benzimidazole small molecules. The different structures of BPS and BPS2 reflect in their solution processibility for film formation and resulting surface energy properties. The presence of alkyl and methoxy chains in BPS2 allow for high-quality films from chlorine-free solvents and improved wettability for the polar solvents typically used for perovskite precursors. BPS possesses similar electrochemical properties to BPS2 but its relatively poor wettability towards perovskite precursor solutions restricts the application of such materials in PeLEDs.

Compared to reference PeLED structures, when BPS2 is incorporated as an interlayer the maximum EQE increased significantly due to improved perovskite film formation and a reduced hole injection barrier. A noticeable enhancement in the maximum luminance from $960 \mathrm{~cd} \mathrm{~m}^{-2}$ to $1193 \mathrm{~cd} \mathrm{~m}^{-2}$ was also observed. These promising preliminary results suggest BPS2 interlayers may be usefully deployed in Pe-LEDs to help reduce the charge injection barrier and improve the perovskite layer processibility during device fabrication. 


\section{REFERENCES}

[1] Van Le, Q., Jang, H.W., and Kim, S.Y. Recent Advances toward High-Efficiency Halide Perovskite LightEmitting Diodes: Review and Perspective. Small Methods, 2018. 2(10): p. 1700419.

[2] Chaudhry, M.U., Wang, N., Tetzner, K., Seitkhan, A., Miao, Y., Sun, Y., Petty, M.C., Anthopoulos, T.D., Wang, J., and Bradley, D.D.C. Light emitting transistors based on solution processed heterostructures of self-organized multiple quantum well perovskite and metal-oxide semiconductors, Adv. Electron. Mater. (2019), 5, 1800985. [3] Kumawat, N. K., Liu, X.-K., and Kabra, D., and Kumawat, N. K., Blue perovskite light-emitting diodes: progress, challenges and future directions. Nanoscale, 2019. 11(5): p. 2109-2120.

[4] Luo, M., Jiang, Y., He, T., and Yuana, M., Metal halide perovskites for blue light emitting materials. APL Materials, 2020. 8(4): p. 040907.

[5] Huang, J., Miller, P. F., Wilson, J. S., De Mello, A. J., De Mello, J. C., and Bradley, D. D. C. Investigation of the Effects of Doping and Post-Deposition Treatments on the Conductivity, Morphology, and Work Function of Poly(3,4- ethylenedioxythiophene)/Poly(styrene sulfonate) Films. Advanced Functional Materials, 2005. 15(2): p. 290-296.

[6] Dong, J., Song, D., Meng, J., Lu, Y., Li, Y., Qiao, B., Zhao, S., and Xu, Z. Interface energy level alignment and improved film quality with a hydrophilic polymer interlayer to improve the device efficiency and stability of allinorganic halide perovskite light-emitting diodes. Journal of Materials Chemistry C, 2020. 8(20): p. 6743-6748. [7] Claudia, M. C., Li, W., Kaifer, A. E., Stockdale, D., and Bazan, G.C., Electrochemical Considerations for Determining Absolute Frontier Orbital Energy Levels of Conjugated Polymers for Solar Cell Applications, Adv. Mater. 2011, 23, 2367-237.

[8] Sonigara, K.K.,Vaghasiya, J.V., Prasad, J., Machhi, H.K., Ansari, M.S., Qureshi, M., and Soni, S.S., Augmentation in photocurrent through organic ionic plastic crystals as an efficient redox mediator for solid-state mesoscopic photovoltaic devices, Sustainable Energy Fuels, 2021,5, 1466-1476. 